\title{
EFFECT OF CURING MODE ON MARGINAL ADAPTATION OF LOW SHRINK COMPOSITE RESIN
}

\author{
Hisham O. Mohammed*, Mohammed T. Fayed**, Nady I. Mohammed ${ }^{* * *}$ and Mohammed A. Masoud M $^{* * *}$
}

\begin{abstract}
Objective The aim of the present study was to evaluate the effect of curing mode on marginal adaptation of low shrink resin composite resin under objectives of two different resin composites, two different curing modes, two different cavity designs and three different storage times.
\end{abstract}

Materials and Methods One hundred and twenty freshly extracted posterior teeth for tested material (silorane and kalore resin composite). Samples were light cured with two light curing units; quartz tungsten halogen (QTH) and light emitting diodes (LED). Microleakage test was measured on top and bottom surface of each sample. Statistical analysis was done using two ways ANOVA.

Results There was a significant influence of the materials and their interaction with LCU on the microleakage value (P0.05<), silorane resins composite have better marginal adaptation to tooth structure than kalore resin composite. LED light cure give better marginal adaptation than QTH light cure.

Conclusion Marginal adaptation improved by using silorane than other formulation used. Elipar light curing source improve sufficient depth of cure in cavity depth at $5 \mathrm{~mm}$. Both the restorative materials tested in the study had significant difference microleakage especially along the gingival seat in class II cavity.

\section{INTRODUCTION}

The use of resin composite for direct restorations in anterior and posterior teeth has increased significantly due to the esthetic demands of patients and concerns regarding mercury in amalgam fillings. Because resin composite requires minimally invasive preparation used to preserve tooth structure and provide natural appearance results. Resin composite also may eventually replace silver amalgam for direct restorations ${ }^{(1)}$.

The greatest limitations in the use of resin composite as a posterior restorative material seems to be shrinkage during polymerization, which leads to poor marginal seal, marginal staining, recurrent caries and post operative hypersensitivity hypothesis ${ }^{(2)}$.
Recently, a silorane based composite was introduced containing cationic ring-opening oxirane monomers, a compensating mechanism for shrinkage stress occurring during polymerization, this new monomer system, called silorane, was obtained from the reaction of oxirane and siloxane molecules which have open rings that compensate polymerization shrinkage during setting reaction ${ }^{(3)}$.

The new kalore resin composite system is based on a new monomer technology from Dupont which utilizes a DX511 molecule in its matrix. This monomer has a long rigid core helps reduce polymerization shrinkage, and two flexible arms helps increase monomer reactivity, it has high molecular weight and low number of carbon double bonds $(\mathrm{C}=\mathrm{C})$ that help reduce polymerization shrinkage and better marginal adaptation

\footnotetext{
* Dentist, Ministry of Health

** Professor of Operative Dentistry, Faculty of Dental Medicine, Al-Azhar University, Cairo, Boys

*** Assistant Professor of Operative Dentistry, Faculty of Dental Medicine, Al-Azhar University, Cairo, Boys
} 
performance than conventional methacrylate based composite restorations ${ }^{(4,5)}$.

Quartz tungsten halogen (QTH) lamps have been the typical light source for dental curing lights for many years. But have many problems. So, to overcome the problems inherent to the halogen curing units, this fairly cost-effective light source is being challenged by the use of the light emitting diodes (LED). In contrast to halogen lamps, light emitting diodes curing units do not produce visible light by heating of metal filament, but by quantummechanical effects ${ }^{(6)}$.

LED curing lights are said to offer the highest photopolymerization productivity. Unlike the halogen curing system, LED curing units give off a narrow spectrum of light. This light falls within the absorption range of photo-initiator camphoroquinone (CQ), resulting in high-energy performance of curing light. This can be a problem with composites using other initiators; therefore, some manufacturers have added an additional LED wavelength to allow for the curing of all composites ${ }^{(7)}$.

Marginal adaptation was defined as the degree of proximity and interlocking of a filling material to the cavity wall, excellent marginal adaptation depends on the quality of the resin composite restorations and adhesive systems. The growth of bacteria on the resin composite surfaces, secondary caries and pulp damage or marginal imperfections may occur at the site of gap formation ${ }^{(8)}$. The origin of marginal leakage is very complex because of the numerous factors contributing to it, polymerization of resin composite produces shrinkage and subsequent water sorption does produce expansions, other factors like coefficient of thermal expansion and marginal adaptation contribute as well, the bond strength of most resin composite is less than the concentration forces; this causes a space that developed between the restoration and enamel wall. This space or gap can be expected and frequently detected on the proximal gingival margins of class II restorations that identified as microleakage ${ }^{(9)}$.
The aim of this study was directed to investigate the effect of curing mode on polymerization shrinkage and their effect on marginal adaptation.

\section{MATERIALS AND METHODS}

Two types of resin composite (silorane resin composite with silorane system adhesive and kalore resin composite with G-aenial bond), Two types of light cure source (Elipar LED and Coxo QTH) were used in this study

A total number of one hundred and twenty freshly extracted non carious human molars due to pathological diseases collected from surgical department of $\mathrm{Al}$ Azhar University, the teeth free of cracks and any developmental defects were used in this study. The teeth were washed under running water to remove blood, scaled with periodontal scaler to remove calculus and remnants of periodontal tissue. Teeth were stored in distilled water at $37^{\circ}$ until use. The distilled water was changed daily.

The teeth were randomly divided into two main equal groups of (60 each), according to cavity preparation design $(\mathrm{CP})$ that was prepared, either standardized class II cavities preparation with gingival seat $3 \mathrm{mmg}$ depth (CP1) or standardized class II cavity preparation with gingival seat $5 \mathrm{~mm}$ depth (CP2). Each main group was sub-divided into two subgroups of teeth (30 each), according to the type of restorative material (RM) that was used, either Silorane resin composite (SC) or Kalore resin composite $(\mathrm{KC})$. These groups were further subdivided into two groups of teeth (15 each), according to the light curing mode (LC) either Quartz tungsten halogen (QTH) or Light emitting diodes (LED). Each least group was further divided according to the storage time (S) into three smaller groups of teeth (5 each), either stored for one day (S1), three months (S2) or six months (S3).

A two standardized design class II cavity was prepared on the proximal surface (mesial or distal) of each tooth. The dimension of the cavity was bucco-lingual width $4 \mathrm{~mm}$, axial wall depth $2 \mathrm{~mm}$, 
the only variation between the two standardized form, the location of the gingival seat from the marginal ridge, one of them at $3 \mathrm{~mm}$ depth (CP1) and the other was at $5 \mathrm{~mm}$ depth (CP2).

After completed of cavity preparation the cavity was cleaned by washing it with water and drying it with oil free compressed air, the teeth were filled after placement of matrix band attached to matrix holder to simulate clinical situation during restoration placement and to avoid polymerization of the restoration from the proximal aspect, then applied the restorative material according to the manufacture instructions.

Each specimen of each group was cured with light cure according to curing mode either QTH light cure or LED light cure with contact distance as close as possible from occlusal aspect according to the manufactures instructions.

The specimens were stored in distilled water at $37^{\circ} \mathrm{C}$ for different storage time ( 24 hours, 3 months, 6 months) until the marginal adaptation testing was performed. Through the period of each storage time the specimens were thermocycled for 1000 cycles in $5^{\circ} \mathrm{C} / 55^{\circ} \mathrm{C}$ with a dwell time of 30 second and a transfer time of 10 seconds.

At the end of periods of storage time of each group the teeth were removed from water and dried with oil free compressed air. Then a soft brush was used to coat the crown and root of each tooth with clear nail varnish except for the restoration and away one millimeter all around the margins of the cavity, the nail varnish was left to dry completely then second layer of varnish was applied to ensure complete sealing of all surface and left to dry completely.

After sealing of the restored tooth, they were immersed in $2 \%$ methylene blue dye solution for 12 hours at room temperature.

The teeth were mounted into special holding device for sectioning. Teeth were sectioned longitudinally in mesio-distal direction through the middle of the restoration using a diamond saw at low speed, during sectioning; water coolant was used.

Both tooth halves were examined under stereomicroscope at $\times 25$ magnifications and photographs of specimens were taken by a digital camera connected to the stereomicroscope. For each tooth halves, the extent of leakage at the occlusal and the gingival margins were evaluated and the microleakage was assessed by scoring the degree of dye penetration in the tooth restoration interface according to the following criteria

Score $0=$ no dye penetration .

Score $1=$ dye penetration along enamel wall only.

Score $2=$ dye penetration along enamel and dentin wall but not reach axial wall.

Score $3=$ dye penetration reaching axial wall.

The results of dye penetration test were collected, assessed and tabulated separately for occlusal and gingival margins and statistical analysis using Kruskal-Wallis, Bonferroni and Wilcoxon signed ranks test. The level of significance was set at $(\mathrm{P}<0.05)$

\section{RESULTS}

Data analysis was performed in several steps. Initially, descriptive statistics for each group results. Multi-factorial analysis of variance ANOVA test of significance was used for comparing variables affecting leakage score mean values (cavity design, resin composite, curing mode and storage time). Non-parametric ANOVA tests were performed to detect significance between storage subgroups. Pairwise Mann-Whitney test was done between cavity, composite and curing lamp). Statistical analysis was performed using Assistant 7.6 statistics software for Windows (Campina Grande, Paraiba state, Brazil). $P$ values $\leq 0.05$ are considered to be statistically significant in all tests. The mean values of leakage (score) for both cavity designs as function of resin composite. 


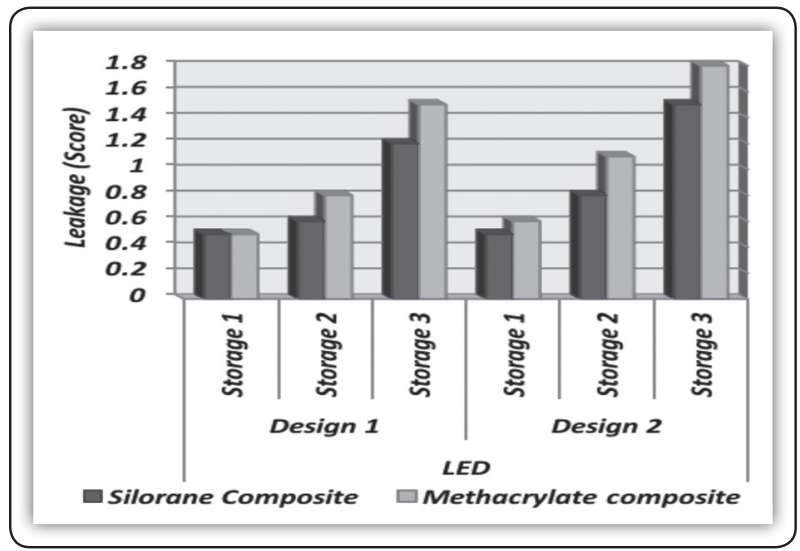

FIG (1) Bar chart of leakage score mean values for both cavity designs comparing between composites as function of storage time with LED curing lamp type

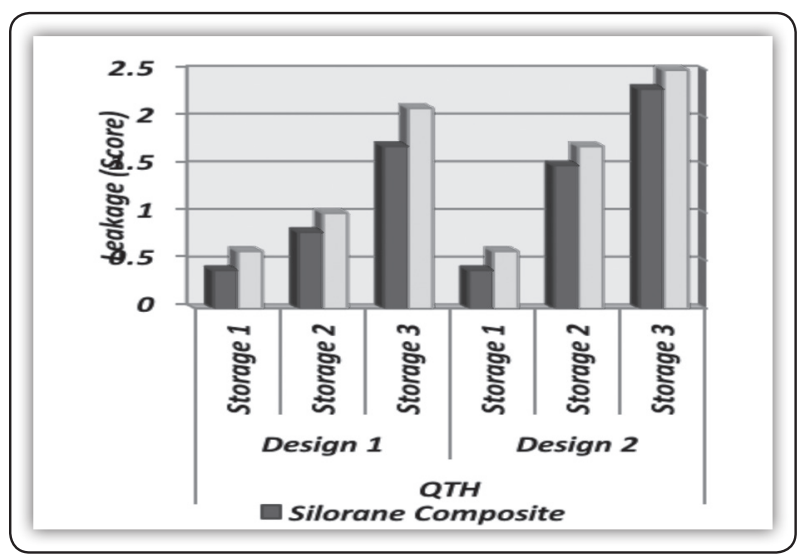

FIG (2) Bar chart of leakage score mean values for both cavity designs comparing between composites as function of storage time with QTH curing lamp type

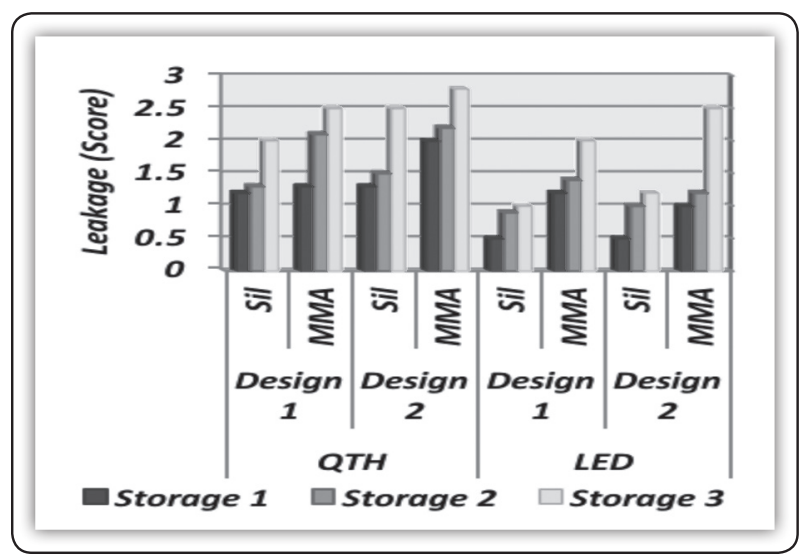

FIG (3) Bar chart of leakage score mean values for both cavity designs as function of composite, curing lamp type and storage time
The results of the study revealed that all the resin materials used with the corresponding adhesive system did not completely eliminate microleakage. The silorane composite have better marginal adaptation to tooth structure than the kalore composite. Also, the gingival margin has more marginal leakage than the occlusal margin. Elipar light cure give better marginal seal between the tooth structure and the restoration at the gingival margin than the quartz tungsten halogen. All restoration achieves highest marginal adaptation after one day of storage. The marginal leakage of all resin material increases with storage time.

\section{DISCUSSION}

Microleakage evaluation is the most common method of assessing the sealing efficiency of a restorative material. Since there is no gold standard for this method, we used 2\% Methylene Blue for 12 hours at room temperature as was previously used by Ernst cp et al in $2008^{(10)}$ who concluded that this immersion period in this concentration had a good correlation with the marginal gaps evaluated using Scanning stereomicroscope connected to digital camera.

Our study concluded that none of the restorative systems tested totally prevented microleakage, the low-shrink silorane system had significantly lower microleakage than kalore system.

The current study revealed that kalore exhibited highest microleakage than silorane. This may be due to the inherent ring opening polymerization of the silorane monomers which can compensate the volume reduction as the molecules come closer to each other compared to the polymerization of the other metharylate one, which is liner polymerization, manifested as a reduction in polymerization shrinkage stress at the tooth/restoration interface ${ }^{(11)}$.

This agrees with Tabassom $\mathrm{H}$ et al in $2012^{(12)}$ and Ilie $\mathrm{N}$ et al in $2009^{(13)}$ who found that microleakage of Silorane was significantly lower than metharylate resin composite restoration. 
The results of this study show that the silorane had significantly lesser microleakage after thermocycling than that of methacrylate. This may be due silorane withstand thermocycling fatigue due to its opening ring silorane chemistry ${ }^{(14)}$. This agrees with Yamazaki PC et al in 2006 ${ }^{(15)}$ and Bagis YH et al in $2009^{(16)}$ who had proved that the microleakage of silorane is lower than that of methacrylate-based composite after thermocycling loading. Studies suggest that thermocycling could accelerate deterioration of the dentin/restoration interface.

Silorane show less microleakage than methacrylate could be due to methacrylate composite is hydrophilic wish lead to water sorption and collagen fiber degradation by time, Silorane consists of 2 molecules: siloxane, which makes the material hydrophobic, and oxirane, which allows polymerization. According to the manufacturer, the polymerization mechanism results in less than $1 \%$ shrinkage. This reduces polymerization stress and, hence, the incidence of microleakage. This agrees with Vanessa et al in 2014 ${ }^{(17)}$

Another explanation silorane showed better sealing ability than methacrylate both above and below the CEJ. This is in agreement with studies of Bagis $\mathrm{YH}$ et al in $2009^{(18)}$ who compared Silorane with nanohybrid composite (Grandio) and found Silorane-based microhybrid composite to have no microleakage. Papadogiannis D et al in 2009(19) also reported that Silorane material showed better behavior than dimethacrylate materials in setting shrinkage and marginal adaptation, this may be due to difference in filler loading or filler size.

Another explanation for Microleakage of silorane lower than methacrylate could be due to different methods in application of adhesive system this confirmed with Krifka S et al ${ }^{(20)}$ who found that the silorane-based resin composite evaluated the best marginal seal.

This finding disagrees with Mohsen $\mathrm{S}$ et al in $2015^{(21)}$ who evaluated Microleakage of Silorane and Methacrylate Based Composite Materials in Class I Restorations; by Using Two Different Bonding Techniques and they found that All the restorative systems tested in this study exhibited microleakage, but the silorane technology showed more microleakage when compared to the methacrylate-based composite systems. This also agreed with Ernst CP et al in $2008^{(22)}$.

The current study revealed that QTH cured subgroup recorded statistically higher leakage score than LED cured subgroup. This may be due to light output intensity of LED higher than the QTH ${ }^{(23)}$.

QTH light cure statistically higher leakage score than LED light cure source, this may be due to LED have high intensity uses a substantially larger semiconductor, which increases both the illuminated area and light intensity more than QTH which enabling a 50\% reduction in curing time, LED present a specific pattern of light emission, which is similar to the absorption spectrum of the camphoroquinone photoinitiator of resin composites, this agree with Thomé $\mathrm{T}$ et al in $2007^{(24)}$ and Bhalla $\mathrm{M}$ et al in $2012^{(25)}$,who found that composites cured with halogen show higher microleakage than composite cured with LED.

Also, the results of this study obtained by Lawrence et al in $2008^{(26)}$ who concluded that the low shrinkage rates produced by the LED due to its low irradiance and low heat generation. The LED produced lower shrinkage values than QTH in conventional and pulse delay modes. This result can be explained by the greater scattering of light by the submicron filler particles than other lightcured resins, requiring more energy for adequate polymerization. This agrees with Fleming GJ et al in $2007^{(27)}$

Also the results with Ernst CP et al in 2004(28) who concluded that Application of the LED curing devices showed a curing potential equal to the QTH when given $40 \mathrm{~s}$ of exposure time, this may be due to wavelength range is already adjusted by filters, this agree with Amaral $\mathrm{cm}$ et al in 2005 ${ }^{(29)}$ 
Another explanation concluded that the photoactivation systems might have no effect on the microleakage of the restorative system used, this confirmed with Cavalcante LM et al in $2007^{(30)}$. This may be due to composite resin leakage is material-dependent.

This finding disagrees with Sharma et al in 2011 (31) who studied Comparative evaluation of marginal adaptation between nanocomposites and microhybrid composites exposed to two light cure units. And they found that least microleakage in microhybrid composites exposed to QTH and maximum microleakage in nanocomposites exposed to LED.

The current study revealed that cavity design2 group recorded statistically higher leakage score than cavity design1 group. Groups that filled with silorane in cavity design 1 show the lowest microleakage while groups that filled with metharylate in cavity design 2 show the highest microleakage.

Increasing in cavity preparation depth increase the possibility of microleakage, this may be due to initiation process which formed by the fragmentation of the photoinitiator will not activated due to insufficient blue light can reach the cavity depth lead to incomplete polymerization of resin monomers occurs, they can be washed out, leaving spaces in the polymeric matrix, which becomes soaked with water, and thus facilitates degradation, this agree with Poonam B et al in 2012(32)

Anotherexplanation concluded that microleakage may be occurred in gingival margin in deep cavities significantly influenced by filling of composite thickness layer, this confirmed with Simos $\mathrm{S}$ et al in 2011 (33) who found that Most manufacturers recommend placing composite in increments of no more than $2.0 \mathrm{~mm}$ in order to eliminate the risk of increased microleakage, An inadequate depth of cure can result in uncured composite, incomplete bonding, and a decrease in marginal seal of the restoration, resulting in microleakage.
The current study revealed that Storage 3 subgroup recorded the highest statistically microleakage followed by Storage 2 subgroup while Storage 1 subgroup recorded the lowest microleakage.

The results of this study showed that the microleakages of all adhesives materials after 3 months and 6 months' time with greater increase especially after 6 months. This might be due to hydrolytic degradation of the resin and collagen fibers in the submicron spaces of the hybrid layer increase with increased exposure to water ${ }^{(34)}$.

Microleakage increase by water storage time may be due to incomplete polymerization of resin monomers occurs, they can be washed out, leaving spaces in the polymeric matrix, which becomes soaked with water, and thus facilitates degradation, this agree with Kitasako $\mathrm{Y}$ et al in 2012 $2^{(35)}$

During long-term water storage, the resin absorbs significant amount of water and consequently swelling of the resin may result in the closure of any space between the bonding resin and dentin surface. Conversely, stresses my simultaneously be induced at the bonding resin-dentin interface, which may pull the collagen fibers into the hybrid layer and resin, leading to tearing along the bonded interface as the collagen fibers become weaker over time from hydrolysis. The increase storage period allows increase water uptake, that lead to increased permeability and increase the hydrolytic degradation of the material. This agrees with Li HP et al in $2011^{(36)}$ who evaluated the effect of longterm storage on nanoleakage

\section{CONCLUSION}

Under the circumstances of this study, the following conclusions were suggested:

1. Marginal adaptation improved by using silorane than other formulation used.

2. Elipar light curing source improve sufficient depth of cure in cavity depth at $5 \mathrm{~mm}$. 
3. Both the restorative materials tested in the study had significant difference microleakage especially along the gingival seat in class II cavity.

4. Storage in water for long periods has a highly significant adverse effect on the marginal seal of all tested materials.

\section{REFERENCES}

1. Keith HS, Chan, Yanjie M, Harry K, Keith C, Desmond N and Jimmy CM (2010): Resin Composite Filling. Materials J (3) 1228-43.

2. Deniz C, John and Burgess (2007): Polymerization shrinkage. Inside Dentistry J (3) 32-6

3. Janaina Bd, Priscila J, Andrea AA, Vinícius DH, Roberta CA, and Paulo HP (2012): Hardness, polymerization depth, and internal adaptation of Class II silorane composite restorations as a function of polymerization protocol. Eur $\mathrm{J}$ Dent (2) $133-40$

4. Douglas A, Terry, Karl F, Leinfelder 2, Markus B and Blatz (2014): A comparison of advanced resin monomer technologies. International Dentistry (6) 50-52.

5. Sensi LG, Strassler HR and Webley W (2007): Direct composite resins. Inside Dentistry J (7) 76-8.

6. Martin FE (2012): A survey of the efficiency of visible light curing units. J Dent (6) 239-45

7. Mahn E (2011): Light polymerization. Inside Dentistry (2) 66-73.

8. Pazinatto FB, Campos BB, Costa LC and Atta MT (2013): Effect of the number of thermocycles on microleakage of resin composite restorations. Pesqui Odontol Bras (17) $337-41$.

9. Ruchi Dhir Sharma, Jaideep Sharma and Anuradha Rani (2011): Comparative evaluation of marginal adaptation between nanocomposites and microhybrid composites. Indian Journal of Dental Research (22) 495-505.

10. Ernst CP, Galler P, Willershausen B and Haller B (2008): Marginal integrity of class $V$ restorations: SEM versus dye penetration. Dent Mater. (24) 319-27.

11. Ola M and Roula A (2010) Microleakage evaluation of silorane based composite. J Conserv Dent. (3): 152-5.

12. Tabassom H, Alireza K and Negin Tabari (2012): Marginal leakage and microhardness evaluation of low-shrinkage resin-based restorative materials General Dentistry. (3) $46-50$
13. Ilie N and Hickel R (2009): Investigations on mechanical behaviour of dental composites. Clin Oral Investig. (4):427-38.

14. Weinmann W, Thalacker C and Guggenberger R (2005): Silorane in dental composites. Dent Mater. (1):68-74.

15. Yamazaki PC, Bedran-Russo AK, Pereira PN and Wsift EJ (2006): Microleakage evaluation of a new low-shrinkage composite restorative material. Oper Dent. (6):670-6.

16. Bagis YH, Baltacioglu IH and Kahyaogullari S (2009): Comparing microleakage and the layering methods of silorane-based resin composite in wide Class II MOD cavities. Oper Dent. (5):578-85.

17. Vanessa Carla Ruschel, Luiz Narciso Baratieri, Sylvio MonteiroJúnio and Mauro Amaral (2014): SiloraneAdhesiveSystem: A Case. Dent Mater J (3):76-85.

18. Papadogiannis D, Kakaboura A, Palaghias G and Eliades G (2009): Setting characteristics and cavity adaptation of low -shrinking resin composites. Dent Mater J. (25) $1509-16$.

19. Krifka S, Federlin M, Hiller KA and Schmalz G (2012): Microleakage of silorane- and methacrylate-based class V composite restorations. Clin Oral Investig J. (4):1117-24.

20. Mohsen S, Alshetili1, Sultan S and Aldeyab2 (2015): Evaluation of Microleakage of Silorane and Methacrylate Based Composite Materials in Class I Restorations by Using Two Different Bonding Techniques. Journal of International Oral Health (2):1-4

21. Hubbezoglu I, Bolayir G, Dogan OM, Dogan A, Ozer A and Bek B (2007): Microhardness evaluation of resin composites polymerized by three different light sources. J Dent Mater. (26): 845-53

22. Thomé T, Steagall W Jr, Tachibana A, Braga SR and Turbino ML (2007): Influence of the distance of the curing light source and composite shade on hardness of two composites. J Appl Oral Sci. (15) 486- 91.

23. Bhalla M, RM Mallikarjuna, TM Nalawade, D Patel, HK Reddy, ND Shashikiran (2012): Effect of light-emitting diode and halogen light curing on the micro-hardness of dental composite and resin-modified glass ionomer cement: An in vitro study. Journal of Indian Society of Pedodontics and Preventive Dentistry. (3) 201-5.

24. Lawrence G, Eduardo Batista Franco and José Carlos Pereira (2008); Effect of light-curing units and activation mode on polymerization shrinkage and shrinkage stress of composite resins. Journal of Applied Oral Science. (4) 1678-765. 
25. Fleming GJ, khan S, Afzal O, Palin WM and Burke FJ (2007): Investigation of polymerization shrinkage strain, associated cuspal movement and microleakage of MOD cavities restored incrementally with resin-based composite using an LED light curing unit. J Dent. (35)97-103.

26. Ernst CP, Meyer GR, Müller J, Stender E, Ahlers MO and Willershausern B. (2004): Depth of cure of LED vs QTH light-curing devices at a distance of $7 \mathrm{~mm}$. J Adhes Dent.(2) 141-50.

27. Amaral CM, Cavalcante LMA, Peris AR, Ambrosano GMB and Pimenta LAF (2005): Microleakage evaluation of resin composite restorations polymerized with different blue light-emitting diode units (LED). Cienc Odontol Bras J. (2) 19-24.

28. Cavalcante LM, Peris AR, Ambrosano GM, Ritter AV and Pimenta LA (2007): Effect of photoactivation systems and resin composites on the microleakage of esthetic restorations. Dent Pract. (2):70-9.

29. Simos S (2011): Direct composite resin restorations: placement strategies. Dent Today J. (8):108-11.

30. Leo Tjäderhane, Fabio D. Nascimento and Lorenzo Breschi (2013): Optimizing dentin bond durability: strategies to prevent hydrolytic degradation of the hybrid layer.Dent Mater. (10): 999-1011.

31. Kitasako Y, Burrow MF, Nikaido T and Tagami J (2012): Long-term tensile bond durability of two different 4-META containing resin cements to dentin. Dent Mater. (18) 276-80.

32. Li HP, Burrow MF and Tyas MJ (2011): The effect of longterm storage on nanoleakage. Oper Dent. (26) 609-16.

33. Hashimoto M, Ohno H, Endo K, Kaga M, and Oguchi $H$ (2005): The effect of hybrid layer thickness on bond strength: demineralized. Dent Mater. (6) 406-11.

34. Venturini D, Cenci MS, Demarco FF, Camacho GB and Powers JM (2006): Effect of polishing techniques and time on surface roughness, hardness and microleakage of resin composite restorations. Oper Dent. (1) 11-7.

35. Al-Wakeel Eel S and Mahmoud SH (2011): Marginal adaptation of ormocer-, silorane-, and methacrylate-based composite restorative systems bonded to dentin cavities after water storage. Quintessence Int J. (10) 131-9.

36. Camps J, Baudry X, Bordes V, Dejou J, Pignoly C and Ladeque P (2006): Influence of tooth cryopreservation and storage time on microleakage. Dent Mater. (2) 121-6. 